\title{
Severity Code
}

National Cancer Institute

\section{Source}

National Cancer Institute. Severity Code. NCI Thesaurus. Code C93670.

A coded value specifying the intensity of an event. 